\title{
SOCIODEMOGRAPHIC CHARACTERISTICS OF PATIENTS USING AESTHETIC PROCEDURES IN CENTERS FOR AESTHETIC MEDICINE
}

\author{
M. Mihailov*, A. Velkova \\ Department of Social Medicine, Faculty of Public Health, Medical University - Sofia, Bulgaria
}

\begin{abstract}
In recent years demand and supply of aesthetic procedures has increased several times. Strengthening of market competition poses new perspectives and challenges to the development of centres for aesthetic medicine. Achieving competitive advantage at the market requires health managers to understand sociodemographic profile of patients, who use aesthetic procedures and to foresee their needs. PURPOSE: The objective of the study was to determine the social and demographic characteristics of patients, using aesthetic procedures. METHODS: We conducted a retrospective study of 712 patients, who underwent aesthetical procedures in a Centre for aesthetic medicine in the period April 2016 - April 2017. There is an increase of aesthetical procedures among males at age below 35. The Centre is located in Sofia and is a newly opened branch of a well-established chain of aesthetic clinics in the country. The information was elicit from the personal patient records in the Centre by a questionnaire. We studied the demographic and social characteristics of the patients, the time and causes of the first visit at the Centre, the type and sequence of the procedures applied. RESULTS: Aesthetic procedures represent $76 \%$ of all activity of the Centre. The typical patient using aesthetical procedures is a woman from Sofia, in the age group 26-55 years. The peak of aesthetic procedures was found to be in the period March - May, with 14.5\% of patent visiting the centre in March for having their first procedure. The most frequent aesthetic procedures were laser epilation and fillers in the facial region. CONCLUSIONS: Understanding the socio-demographic profile of the patients of Centres of aesthetical medicine will help in precise identification of target groups, in development of service packages adequate to the age and aesthetic needs of the patients and in implementation of effective marketing strategies that guarantee the attainment of the health and profit goals of the organization.
\end{abstract}

Key words: aesthetic dermatology, cosmetic dermatology

\section{INTRODUCTION}

In recent years aesthetic procedures are becoming more and more popular. Supply and demand of similar services has increased many fold as a result of numerous factors. Longlasting beauty, attractiveness and youth are established norms of the modern society, which dominate the cultural development process and are supported by media, industry and institutions (5). Developments in society, technology and medicine have caused the creation of a new branch of medicine aesthetic medicine, which quickly and convincingly finds its healers and customers. Even though there is still no widely accepted and valid description defining and outlining the

\footnotetext{
*Correspondence to: Mihail Mihailov, Department of Social Medicine, Phaculty of Public Health, 8 Bialo More Str.,

Sofia 1527, Phone: +359878371665, e-mail: mihail.p.mihailov@gmail.com
}

limits of the specialty, it is perceived as a "specialty made out of procedures, which aim to improve the outer appearance and satisfy patients by using noninvasive and minimally invasive cosmetic procedures" (7).

Patients looking to improve their appearance are motivated by different reasons. Some of them look for an improvement in the aesthetic of the face and body driven by a desire to achieve the perfect form; others are fighting the signs of the inevitable aging; while another group is restoring their aesthetic appearance and/or functioning after severe burns, injuries or diseases (2).The users of aesthetic services are faced with a long list of available therapies. Most of them are connected to care and rejuvenation of the skin, decreasing the visible imperfections and wrinkles, treatment of scars, lip augmentation, muscle relaxation, removal of pigmentations, tattoos and capillaries, non- 
surgical lifting of the face, removal of excess fat formations, mesotherapy, and others (8).

The increased demand for aesthetic procedures has caused the number of medical centers that offer them to also increase. The growing competition in the market of aesthetic services creates new perspectives and challenges for the development of aesthetic medicine centers (6). Achieving a competitive advantage is possible when the medical managers working in the centers know the sociodemographic characteristics of their patients well and proactively prepare for their needs (3).

\section{AIM}

The study aims to identify the sociodemographic profile of the patients using aesthetic procedures.

\section{MATERIALS AND METHODS}

A retrospective descriptive study was conducted based on the medical documentation of 712 patients, who had aesthetic procedures done in Center for Aesthetic Medicine (CEM) in the period between April 2016 and April
MIHAILOV M., et al. 2017. The aesthetic center is situated in Sofia and is a new branch of a well-known chain of aesthetic medicine centers in the country. All customer visits during the period of the study were identified from the customer information database of the Center. We analized the records of patients who had aesthetic procedures done. The primary information is extracted from the individual health records of the customers in the Center using a questionnaire. The demographic and social characteristics of the individuals were studies, as well as the time and reason for the first visit, the type, sequence and frequency of aesthetic procedures.

\section{RESULTS}

Aesthetic procedures accounted for $3 / 4$ of all realized appointments in the Center for Aesthetic Medicine during the period of the study (Figure 1). The center was also recognized by the customers as a place to receive a dermatological examination and consultation on aesthetic aspects of different medical problems.

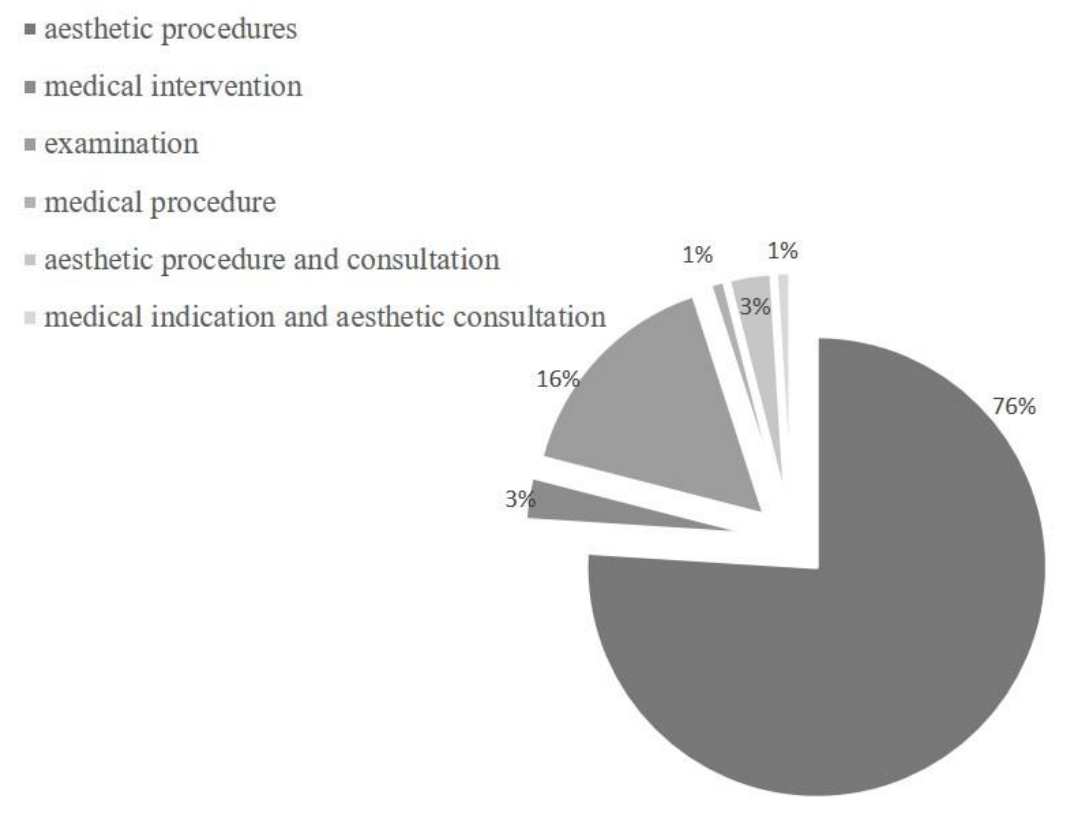

Figure 1. Reasons for visiting the Center for Aesthetic Medicine (in percent)

As expected, women were the main customers of aesthetic medicine and accounted for $88 \%$ of the patients who had such a treatment for one year in the Center. The typical patient, served in the Center, can be described as a woman from Sofia with median age $36,3 \pm$ 10,8 years. The underlying causes that explain the observed gender distribution are: traditionally, woman are more likely to subject themselves to procedures in the name of beauty, as they strive to look attractive and these behaviors are socially accepted and approved; aesthetic procedures accentuate femininity and help achieve a more youthful appearance, which aligns with the personal desires of most women (to overcome the visible effects of aging). The effect of customer segmentation and targeting in marketing strategy - offering aesthetic procedures mainly aimed towards women - is also significant (1).

Our results concerning the gender distribution and customers of aesthetic medicines do not significantly differ from those in other countries. For example, the statistics for 2016, presented by the American society for aesthetic and plastic surgery, show that more than 10.5 
million women have subjected themselves to aesthetic nonsurgical procedures (9). Only $8.9 \%$ of the aesthetic procedure customers in the USA are men. They have mainly chosen aesthetic procedures that fight the effects of aging. The most popular procedure is the application of a botullin filler, while the second most popular therapy for men is hyaluronic fillers.

We studied the age distribution of patients who had aesthetic procedures done. It varied greatly - from 7 to 70 (Table 1). The largest group of patients was the one in the age of the largest social activity (26-55 years of age). This is the age of completing education, looking for a partner, achieving professional realization, building of the own social position and other
MIHAILOV M., et al. important events in an individual's life cycle, during which the good appearance can be an advantage. When clustering the patients in two larger age groups, below 35 years of age and over 35 years, it became evident that they were almost equal (with those over 35 accounting for $52.7 \%$ ). The expansion of the age range of aesthetic procedures is connected to the increasing desire of people to preserve their youthful appearance for longer and slow down the visible effects of aging. As the age of retirement is increasing, many more people stay socially active until 70-75 years of age, therefore increasing the number of individuals looking for ,anti-aging” aesthetic interventions to improve appearance.

Table 1. Age structure of the patients of aesthetic procedures

\begin{tabular}{|l|l|}
\hline \multicolumn{1}{|c|}{ Age group } & \multicolumn{1}{|c|}{ Number and percentage } \\
\hline From 7 to 18 years of age & $14(1,96 \%)$ \\
\hline From 19 to 24 years of age & $106(14,88 \%)$ \\
\hline From 25 to 34 years of age & $216(30,33 \%)$ \\
\hline From 35 to 54 years of age & $334(46,91 \%)$ \\
\hline From 55 to 70 years of age & $(5,75 \%)$ \\
\hline
\end{tabular}

In comparison, the American society for aesthetic and plastic surgery found that individuals who use aesthetic procedures most often are between 35 and 50 years old. These patients are $39.3 \%$ or 5.3 millions from all who had aesthetic procedures done in 2016. In USA, the patients 18 and under account for $1.5 \%$ of all aesthetic nonsurgical procedures. The most common procedure in this age group is laser/photo-hair removal. Patients in the age group of 19-34 years old are $16.3 \%$ of the market of aesthetic nonsurgical procedures. The most popular procedure for this group is the application of bottulinum toxin. The group of patients between 35 and 50 years of age have almost the same preferences of the type of aesthetic procedures $-38.8 \%$ of them use aesthetic surgical procedures, while 39.3\% aesthetic nonsurgical procedures. The most frequent and desired procedure for them as well is bottulinum toxin with almost 2 million customers for a year. In second place come hyalluronic fillers with close to 1 million patients. In this age group the results from the aging process become visible and fast results are being sought. Patients between 51 and 64 years old make up $30.8 \%$ of the population using aesthetic procedures. Individuals over the age of 65 account for $12.1 \%$ of all users of aesthetic procedures in the USA.

The comparison between the age distributions of Bulgarian and American patients who had aesthetic procedures done does not show significant differences.

An important demographic characteristics of the users of aesthetic services is their place of living (Figure 2). The patients in our study were mainly residents of Sofia $(76 \%)$. This was a result due to the fact that the Center for Aesthetic Medicine is positioned in the municipality of Sofia. The high population concentration in Sofia - close to $17 \%$ of the total population, mainly in the younger age groups, forms a significant cohort of potential users of aesthetic services. Those residing in the capital also have a significantly better access to aesthetic procedures, not only because of the large amount of aesthetic centers, but also due to the higher median wages per capita, which guarantee a higher purchasing power. According to the National Statistics Institute of the Republic of Bulgaria, the salary of those working in the capital is higher than the median for the country. In the last quarter of 2017 it amounted for approximately 1400 Bulgarian leva.

The second largest group of patients using aesthetic services appeared to be the customers living abroad. The Center for Aesthetic Medicine was attractive to them, among the other factors, due to easily accessible location - close to the airport and a metro station. The 
Center also maintains a well-organised and informative Internet site, available in Bulgarian and in two foreign languages, which is used to invite patients from abroad, for whom the prices of aesthetic procedures in Bulgaria are
MIHAILOV M., et al. attractive and affordable. The high quality of conducting the procedures and the ability of staff to freely communicate in a language the patients speak are additional reasons for the higher number of patients from abroad.

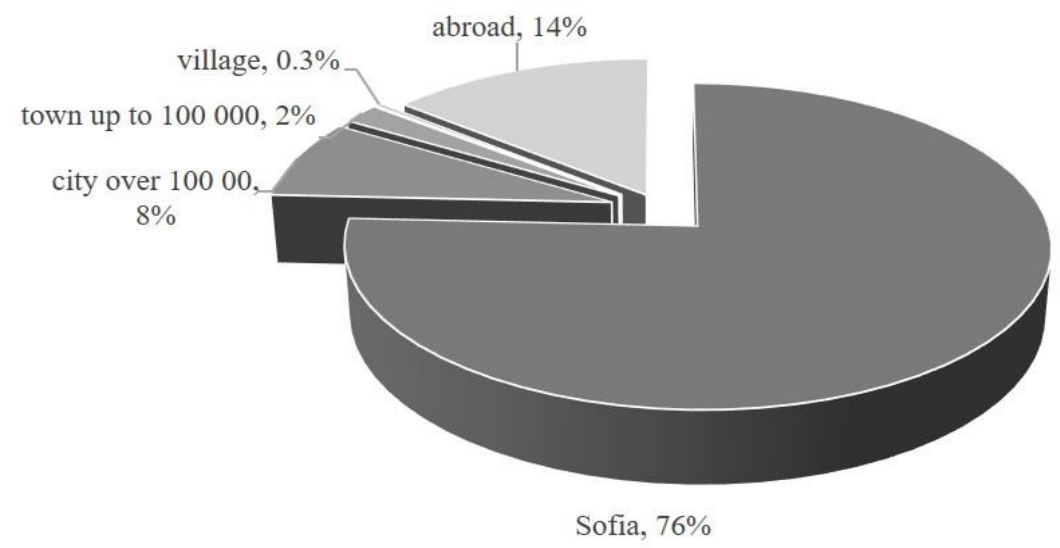

Figure 2. Structure of patients according to place of living

The seasonal distribution of the first visit of patients for an aesthetic procedure was also investigated in the study (Figure 3). The peak of demand for aesthetic procedures was in the period between March and May. The visits in March accounted for $15 \%$ of all realized appointments for aesthetic procedures in the Center for the entire year. In this period of the year, individuals purchase more aesthetic procedures in order to improve their appearance and confidence before the beginning of the summer season, in which social communication is especially intensive.
The improved appearance through a variety of mechanisms positively affects social contacts. Their quality, in turn, positively influences the overall quality of life.

The seasonality in the demand for aesthetic procedures, observed by us, makes it reasonable to recommend health managers in centers of aesthetic medicine to form packaged offerings, which can be conducted in consequent months, when the stream of new patients is smaller. This way, centers can also ensure customers for the less busy months.

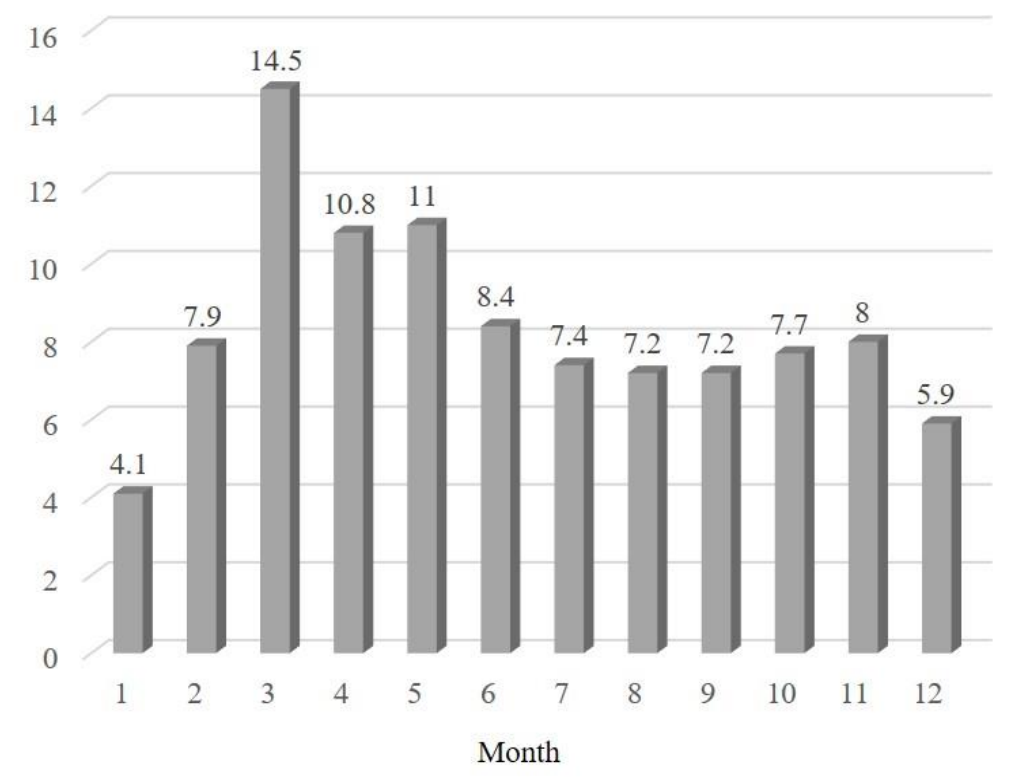

Figure 3. Seasonality of the first visit for aesthetic procedures

As part of the research, a comparative analysis of the patients below and over 35 years of age was conducted, comparing the groups based on gender, place of living, reasons for visiting the 
Center and type of the conducted aesthetic procedures. The gender composition of the two age groups showed insignificant differences. In the group up to 35 years of age, men were $14.5 \%$, while in the other group $-9.9 \%$ $(\mathrm{p}=0.06)$. The growth in demand for aesthetic services from young men (up to 35 years of age), is most likely a result of the formation of a new aesthetic ideal and striving for attractiveness, which overcomes the typical man behavior towards appearance. Young men are looking for noninvasive procedures with a quickly visible effect. Older men still remain conservative in their opinion towards aesthetic medicine. Numerous authors point out that men experience significant health benefits from aesthetic care for the body, and today's society is becoming more and more tolerant towards conducting aesthetic procedures on men. This is a result of the change in the manner by which modern men demonstrate strength and potential to ensure advantages and survival for themselves and their families (4). Using aesthetic procedures is a sign of social advantage, health and adaptability, and is becoming more socially acceptable. Marketing strategies oriented towards men as users of aesthetic procedures require a knowledge and understanding of the drivers of their behavior, as well as an appropriate choice of appropriate promotion tactics.

The comparison between the two age groups by place of residence did not find significant differences. The differences in the reasons for visits at Center for Aesthetic Medicine, however, were significant. In the group up to 35 years of age, every fifth patient realized an appointment for exam or consultation $(21 \%)$, while in the group over 35 years of age, every $8^{\text {th }}$ individual realized a similar appointment $(\mathrm{p}=0.04)$. This shows the improved health behaviour of younger people, who understand the necessity of early and systematic care for the body, including care for the appearance, and its connection to the psychological and social wellbeing.

The comparison of the frequency of conducted aesthetic procedures in the two age groups showed that the individuals up to 35 years of age prefer laser hair removal procedures (they occur twice as much as in the other group); fillers and machine procedures with a regenerative effect aimed at prevention of aging. Patients over 35 years of age preferred procedures with botox; laser ablative procedures, which have a good effect on the skin quality and age spots; fillers for replacing volume in the face. The frequency of application of mesotherapy, boosters,
MIHAILOV M., et al. plasmotherapy and laser non-ablative procedures did not differ significantly between the two groups.

\section{CONCLUSIONS}

Based on our observations and analysis, we have made the following conclusions:

- Aesthetic procedures are a main part of the workload of centers of aesthetic medicine and are popular amongst a particular group of customers;

a The typical patient of the Center for Aesthetic Medicine is a woman with median age 37 years old, living in Sofia.

- The frequency of demand for aesthetic services by young men of age up to 35 is increasing, most likely due to the change in signs, demonstrating physical and social superiority in men and the increase in social acceptance towards aesthetic procedures conducted on men.

- The most appointments for aesthetic procedures (around 35\%) can be seen in the spring months in connection to the upcoming more intensive social contacts during the summer.

- The most frequently conducted aesthetic procedures are laser hair removal and corrections of volumes in the zone of the face with fillers, and in the group of individuals up to 35 years of age procedures aimed at preventing aging are popular.

a The retrospective nature of this study limits the exploration of the effect of other important social characteristics such as education, income, family status, which can specifically affect self-perception and self-esteem, depressiveness and anxiety factors with an important role in forming the decision to seek aesthetic procedures.

Aesthetic procedures are gaining popularity in Bulgarian society. More and more medical centers, which offer these services, are opening their doors. Managers are faced with the reality of a growing competitive market. It is necessary to analyze the activities and outline the perspectives for development of centers for aesthetic medicine. An understanding of the sociodemographic profile of the target groups using aesthetic procedures creates the ability to precisely define the target cluster, develop ageand need-appropriate service packages and implement effective marketing strategies, guaranteeing the achievement of the health and economic goals of the organization. 


\section{REFERENCES}

1. Lewis, W., The symbiotic relationship between aesthetic medicine and social media, Aesthetics, volume 2/issue 7, June 2015.

2. https://www.aaamed.org/aesthetic_med.php

3. Elsaie, M.L., Psychological approach in cosmetic dermatology for optimum patient satisfaction. Indian Journal of Dermatology.; $\quad$ 55(2):127-129. doi:10.4103/0019-5154.62733., 2010.

4. https://www.plasticsurgery.org/news/plastic -surgery-statistics

5. Sachdev, M/ and Britto, G.R., Essential Requirements to Setting up an Aesthetic Practice. Journal of Cutaneous and
MIHAILOV M., et al. Aesthetic Surgery.;7(3):167-169. doi:10.4103/0974-2077.146675, 2014.

6. Goh, C., The need for evidence-based aesthetic dermatology practice. Journal of Cutaneous and Aesthetic Surgery.; 2:65-71, 2009.

7. Enchev, A., Marketing in Plastic Restorative and Aesthetic Surgery. Social medicine, - journals.mu-varna.bg, 2015.

8. https://www.surgery.org/

9. Frucht, C. and Ortiz, A., Nonsurgical cosmetic procedures for men: Trends and technique considerations. Journal of Clinical and Aesthetic dermatology, Vol. 9, num.12, p.33-34, December 2016. 